UNDERGRADUATE RESEARCH IN NATURAL AND CLINICAL SCIENCE AND TECHNOLOGY (URNCST) JOURNAL Read more URNCST Journal articles and submit your own today at: https://www.urncst.com

\title{
Neural Connectivity and Episodic Memory in Autism Spectrum Disorder: A Literature Review
}

\author{
Xalima Ali, BSc Student [1]* \\ [1] Faculty of Health Sciences, University of Waterloo, Waterloo, Ontario, Canada N2L 3G1 \\ *Corresponding Author: xaali@uwaterloo.ca
}

\begin{abstract}
Introduction: There is a growing interest in the social and biological context of episodic memory in children with autism spectrum disorder (ASD). Research has previously found that episodic memory deficits are overrepresented in this population. In an attempt to learn why children with ASD are disproportionately impacted by episodic memory impairments, this paper explores literature from 1970-2020 concerning the relationship between functional connectivity (FC), effective connectivity (EC) and structural connectivity (SC) and episodic memory in children with ASD.

Methods: The method of this review involved an extensive literature search in scientific databases for experimental studies and magnetic resonance imaging (MRI) data pertaining to episodic memory in children with ASD. The literature review was conducted by searching for literature in electronic databases (Google Scholar, PubMed and MEDLINE) using the following search words: "ASD and memory," "episodic memory in ASD," "connectivity in ASD".

Results: In the studies reviewed, children with ASD consistently underperformed on episodic memory tasks relative to typically developing children. Additionally, the MRI scans of the children with ASD showed hyper- and hypoconnectivity of brain regions across the three connectivity metrics. The results indicated that the abnormalities seen in the FC, SC, and EC of children with ASD is an area of research and intervention opportunity for clinicians.

Discussion: Research has found that interventions introduced early to children with autism have the potential to reduce symptoms of ASD before adulthood. Therefore, it is important that early interventions related to improving episodic memory are introduced to children early on to increase quality of life later. Additionally, future research must explore if connectivity abnormalities contribute to ASD or if it precedes ASD diagnosis. As a result, clinicians may also consider adding episodic memory deficits to the diagnostic criteria for ASD since it is overrepresented in this population.

Conclusion: Clarifying the relationship between ASD, connectivity, and episodic memory will improve the quality of life of children with ASD in the future. This understanding will have broader implications in children and adults with ASD who struggle with episodic memory in terms of improving their experience in education, work and personal life.
\end{abstract}

Keywords: autism spectrum disorder; connectivity; episodic memory

\section{Introduction}

The diagnostic criteria for autism spectrum disorder (ASD) from the Diagnostic and Statistical Manual of Mental Disorders (DSM-5) characterizes ASD as the combined difficulties in social communications and interactions, and repetitive and restrictive patterns of behaviours [1]. ASD can be a lifelong disorder and has a wide spectrum of symptom severity impacting speech, cognitive, and social abilities. According to a Centres for Disease Control and Prevention report, the prevalence of ASD has increased substantially in a short period of time, currently estimated to impact 1 in 54 children [2]. Currently, no treatment exists to cure ASD, although interventions are available to reduce symptoms impacting social and cognitive ability. The etiology and risk factors of autism are still being studied; however, most studies agree that genetics are a risk factor. Inconsistency and gaps in autism research are largely due to the developmental differences existing within the diverse ASD population [3].

Clinicians are increasingly using magnetic resonance imaging (MRI) to explore brain connectivity in ASD. An MRI is a non-invasive procedure that uses computergenerated radio waves and magnets to produce detailed images of internal organs and tissues. Brain activity is associated with relative concentrations of oxygen in local blood supply. An increase in activity, is delineated by a increase in oxygenated blood flow. The susceptibility to the powerful magnets of the fMRI differs among oxygenated and deoxygenated blood. fMRI interprets the differences by measuring the blood-oxygen-level dependent (BOLD) response. BOLD signals represent the net increase in blood oxygenation following brain activity. MRI scans are useful as a diagnostic tool for ASD and other developmental abnormalities as clinicians can examine brain volume and 
UNDERGRADUATE RESEARCH IN NATURAL AND CLINICAL SCIENCE AND TECHNOLOGY (URNCST) JOURNAL Read more URNCST Journal articles and submit your own today at: https://www.urncst.com

connectivity before or after the onset of symptoms [4]. Brain connectivity is defined as the pattern of neural anatomy or activity between distinct units within the nervous system. One of these links is the temporal correlation of neural connections referred as functional connectivity (FC). FC is usually measured during resting state fMRI and, in principle, be estimated using any general method for estimating statistical dependencies among time series data. A FC analysis strives to identify the temporal correlation between the changes in BOLD signals between different brain regions. A higher FC would signify stronger temporal correlation between the changes in BOLD signals between different brain regions. fMRI (fMRI) observes FC through the measurement of brain activity to detect abnormalities in the brain's functional anatomy that are costly and difficult with other imaging techniques [4]. Another type of brain connectivity is the neural coupling pattern known as effective connectivity (EC) [5]. EC measures the relationship between human brain regions in terms of their directed influence on each other. Unlike FC, EC measures the effect of one brain region on another in a particular direction, which can provide more insight into the mechanisms underlying brain function, namely how one region influences another. EC can be used to determine which brain regions are exhibiting altered function and influences on other brain regions. An estimated EC measures the strengths of causal interactions within and across brain areas by measuring the changes in BOLD signal during time. A higher EC would demonstrate there is a higher directional influence from one brain region to another. fMRI can also observe effective connectivity in order obtain insight on the performance of brain operations [5]. A third type of connectivity is structural connectivity which refers to the white matter tracts of interconnecting brain regions (SC) [4]. Structural MRI examines the anatomy and pathology of the brain which allows SC to be observed in order to determine if there are irregularities in the structure of the brain [4].A high structural connectivity would indicate there is higher existence of white matter tracts physically connecting brain regions. fMRI scans are used to assess and explore brain activity while structural MRI scans are used to examine the anatomy and pathology of the brain [4].

Although not included in the DSM, there is increasing evidence of ASD being associated with episodic memory deficits [6]. Episodic memory is a category of declarative memory that involves past events and individual experiences, including knowledge of where and what happened [7]. Several studies comparing children with and without ASD found that the children with ASD experienced more difficulty in recalling episodic memories [6].

Since previous studies have found evidence of the association of episodic memory impairments and children with ASD, the aim of this study was to perform a literature review to examine why children with ASD are disproportionately impacted by episodic memory impairments through the analysis of different MRI studies. Specifically, the results of studies using functional and structural MRI will be analyzed in order to explore the relationship between FC, EC, and SC and episodic memory in children with ASD. Learning how memory is impacted in children with ASD will pave the way for future learning or intervention programs for children with ASD. Additionally, with the insights from this study, new research avenues involving the reversal or treatment of deficits in episodic memory in children with ASD can be explored.

\section{Methods}

Research papers dating between 1970-2020 were collected to examine how connectivity impacts episodic memory problems associated with ASD. The literature review was conducted by searching for literature in Google Scholar, PubMed and MEDLINE. Search words that were used to find the studies were the following: "ASD and memory," "episodic memory in ASD," "connectivity in ASD." The inclusion criteria were all academic studies found that explore connectivity in children with ASD using functional and structural MRI data or experimental trials. The exclusion criteria were any studies researching memory types other than episodic memory or studying memory in children without ASD.

\section{Results}

A common finding in studies examining this field of literature is that children with ASD tend to face difficulty and demonstrate poor performance when completing episodic memory tasks [6]. Southwick et al. conducted a study on children from ages 5-19, with and without ASD that tested their memory functioning through a variety of tasks and found that the group with ASD had reduced episodic memory [8]. Researchers have highlighted the growing support from functional neuroimaging that shows that memory impairments in ASD can be explained through investigating connectivity between different regions of the brain [4].

\section{FC and Episodic Memory in Children with ASD}

Most FC studies reviewed had evidence of the importance of FC during episodic memory retrieval. One study used fMRI scans to compare the FC of neural networks in children with ASD to children with no neurological disorders [12]. Comparisons with controls revealed nine networks with similar spatial patterns in these two groups. Children with ASD, in comparison to controls, showed decreased connectivity in the following areas: left gyrus rectus, left middle occipital gyrus, right angular gyrus, right middle frontal gyrus and right inferior frontal gyrus (IFG), orbital part within the lateral visual network, the left IFG, right precuneus, and right angular gyrus within the left frontoparietal network [12]. Further research has found that connectivity in these areas is vital to episodic memory in early childhood [12]. Riggins et al. found that FC in those 
UNDERGRADUATE RESEARCH IN NATURAL AND CLINICAL SCIENCE AND TECHNOLOGY (URNCST) JOURNAL Read more URNCST Journal articles and submit your own today at: https://www.urncst.com

areas was positively associated with episodic memory in children. Contrastingly, a study examining connectivity in children with ASD found that hyperconnectivity was associated with the memory impairments seen in children with ASD. Monk et. al evaluated connectivity in children with ASD and found that children with ASD had hypoconnectivity of the posterior cingulate cortex (PCC) and FG, however, hyperconnectivity was found between the PCC and bilateral temporal lobes and right-hippocampal gyrus [13]. Researchers such as Ozonoff et al. have evidence that suggest severe symptoms associated ASD are a result of memory deficits associated with FC abnormalities [14].

\section{$\underline{\mathrm{SC} \text { and Memory in Children }}$}

In all reviewed studies concerning SC and memory in children with ASD, researchers found a widespread deficit of white matter in the children with ASD. Fitzgerald et al. found white matter abnormalities in the cingulum of children with ASD, a structure in the limbic system involved in episodic memory retrieval requirements such as attention, memory retrieval, and emotional processing [9]. Several of the studies reviewed conducted on children with ASD found abnormalities regarding structural changes in the corpus callosum. The corpus collosum is the largest white matter structure in the brain and is vital for brain communication [9]. Vogan et al. used diffusion tensor imaging, a subtype of MRI neuroimaging, to show white matter differences in a large sample of children with ASD [10]. The researchers found the corpus callosum to be significantly smaller in children with ASD and suggested that the impacts on information processing may be linked to memory and attention impairments [10]. Several studies have found that children with ASD tend to have damage to the corpus collosum and other structures in the brain that may be negatively associated with episodic memory. Another study involved the use of a standardized test and an episodic memory task in order to examine the relationship between the delineated white matter tract and episodic memory in children [11]. The results of both the test and memory task showed poor white matter connectivity between the hippocampus and medial prefrontal cortex along with difficulty in performing an episodic memory task [11]. In their linear regression model, Ngo et al. showed that white matter connecting the hippocampus to the inferior parietal lobule predicts children's performance on episodic memory tasks [11]. Interestingly, although SC abnormalities were found to be prominent in children with ASD, few studies linked these SC impairments and episodic memory impairments found in the children studied as a behavioural phenotype for ASD.

\section{EC and Episodic Memory in Children with ASD}

Although there were much more studies in the review concerning SC and FC, there were a few studies that described how EC underlies episodic memory in children with ASD [5]. One study observing EC to the precuneus and cuneus found decreased EC from the temporal pole to the ventromedial prefrontal cortex, and lower activity in the ventromedial prefrontal cortex [5]. This was consistent across studies. Ren et al. found a combination of increased and decreased EC in areas of the brain relative to controls [15]. Firstly, they found lower EC in the middle temporal gyrus and other temporal areas to the precuneus and cuneus. Secondly, the researchers observed higher EC from the hippocampus and amygdala to the temporal gyrus. Results also showed that the prefrontal cortex areas had higher EC with each other and with the precuneus and cuneus. Lastly, there was decreased EC from the temporal pole to the ventromedial prefrontal cortex. Ren et al. discovered evidence supporting that EC of the anterior hippocampus impacts episodic memory retrieval [15].

\section{Discussion}

These results indicate that episodic memory retrieval functions in ASD are markedly different, and functional connections play an important role in understanding episodic recollection-related retrieval deficits in the ASD children population. The overall findings suggest that children with ASD have decreased connectivity in left hippocampal networks relative to typically developing children, suggesting they may depend more on local memory systems that are related to non-relational learning.

\section{Function Connectivity (FC)}

A possible explanation for the limited successful episodic memory outcomes of few ASD participants in some FC studies is the independent use of the left anterior hippocampus and parietal areas. The (name of model) (BBB) model, a model of XYZ, suggests that the hippocampal region is required to retrieve and construct mental images during environment perception. An image of the perceived environment is then maintained and manipulated by the hippocampal region. This model proposes that episodic memory will always be hippocampus-dependent if it is associated with a rich mental image of its surroundings [16]. Historically, there has been evidence supporting that hypoconnectivity, hyperconnectivity, and or the combination of the two may underlie the episodic memory problems seen in many children with autism [17]. One of the most accepted opinions regarding autism and functional connectivity is that ASD and the memory problems associated with it may result from the combined effects of hypo and hyper functional connectivity [12]. The abnormalities of these functional networks may be indicative of ASD and identifying this functional change may improve understanding of how symptoms related to episodic memory deficits in people with ASD are manifested.

\section{Structural Connectivity (SC)}

Overall, the findings from studies examining $\mathrm{SC}$ demonstrate that children with ASD tend to have poor SC relative to typically developing children. Specifically, there 
UNDERGRADUATE RESEARCH IN NATURAL AND CLINICAL SCIENCE AND TECHNOLOGY (URNCST) JOURNAL Read more URNCST Journal articles and submit your own today at: https://www.urncst.com

may be a weaker association between the white matter tracts connecting specific brain areas and cognitive functions in children with ASD, which may indicate less efficient brain networks than those in typically developing children [9]. Findings showed that children with ASD tend to have smaller a corpus callosum than controls. Previous studies have showed how structural connectivity measures correlate with the size of relevant regions of the corpus callosum. Specifically, a larger corpus callosum is associated with greater structural connectivity of the brain, and in turn, greater episodic memory performance. This would be another possible explanation to the episodic memory impairment and structural connectivity associations [10]. The results also showed that hippocampal-inferior parietal lobe white matter plays an important role in determining episodic memory performance [11]. Compared to other white matter pathways, this pathway showed higher activity when children were able to accurately perform an episodic memory task than (?) [11]. Based on this evidence, it appears these prefrontal areas have altered structural connectivity that may also negatively affect neural communication in children with ASD, and in turn-episodic memory.

\section{Effective Connectivity (EC)}

In terms of EC, previous MRI studies have found that the ventromedial prefrontal cortex plays a crucial role in contextual memory retrieval, memory consolidation, and memory-guided decisions [5]. The results of previous studies strengthen the theory that decreased EC in the ventromedial prefrontal cortex may be associated with episodic memory impairments found in children with ASD [15]. Rather than distinguishing between recollection and familiarity, some researchers proclaim that increased hippocampal EC activity is associated with strong memories and the lower EC found in children with ASD may contribute to weaker episodic memory commonly found in this population [15].

\section{FC, SC, and EC}

All in all, most findings were consistent with earlier literature supporting that episodic memory impairments found within children with ASD are largely due to brain connectivity abnormalities. Decreased SC among ASD children with episodic memory impairments was consistent across studies, however there was a mix between hypo and hyper connectivity among functional and effective brain regions. A direct comparison is necessary to determine whether the present findings of decreased SC in ASD correspond to these functional differences.

\section{Limitations and Strengths}

Characteristics of the sample from the present studies reviewed may limit generalizability. Notably, most participants identified as Caucasian and male. The prevalence ratio of ASD is often reported as 4 boys diagnosed for every 1 girl diagnosed [2]. Children with
ASD tend to be diagnosed with other medical conditions later in their life such as obsessive-compulsive disorder, attention deficit hyperactivity disorder, anxiety, and other health conditions [1]. It is difficult to say with certainty that the episodic memory impairments found are largely due to connectivity abnormalities found in the ASD population or the population of the other health conditions. Additionally, there is uncertainty whether the connectivity problems precede ASD behavioural symptoms or vice-versa. Moreover, the ASD population is so diverse as some people may be non-verbal and have poor cognitive function that it is unknown whether the findings of this study would reflect beyond the verbal high-functioning participants in the studies analyzed in this review. Furthermore, since studies were collected from 1970 to 2020, and there have been major developments in research, diagnosis, treatment, and symptoms of ASD within that time period, it is possible that there could be gaps within the findings that may be addressed in future studies. There is still uncertainty on the differences in the neural development of children with ASD, the genes associated with ASD risk and their function are also yet to be determined.

Neurological conditions such as ASD often manifest itself with difficulties retrieving and processing memories [3]. It is therefore of tremendous clinical importance to understand the neuropathological correlates of memory deficits. However, research progress has been slow in the case of children with ASD [19]. It is partly due to the fact that imaging children requires a level of compliance and patience that even typically developing adults have difficulty meeting. Imaging technologies that accommodate for children with ASD would significantly advance understandings of their altered information processing [19].

A common challenge in research in ASD is that there is a dependence on behaviour rather than biology [19]. This review addressed this challenge by exploring experimental studies and MRI data for information on the impacts of connectivity on episodic memory in children with ASD as well as the episodic memory performance for children with ASD. This increased consistency and reliability of the present results. This review summarizes the findings of functional, structural and effective connectivity studies related to episodic memory in children with ASD independently and together.

\section{Future Studies and Implications}

In light of the general findings for connectivity in children with ASD explored in this present study, future studies must work to determine if these results are generalizable to the diverse profiles of children with ASD. During the period of neuroplastic development, the domains of language, cognitive, and social abilities are developed, and neural integration occurs [20]. Research has found that this developmental window of opportunity is found in ages 0-3 years. In addition to providing children with the best start, early intervention increases the 
UNDERGRADUATE RESEARCH IN NATURAL AND CLINICAL SCIENCE AND TECHNOLOGY (URNCST) JOURNAL Read more URNCST Journal articles and submit your own today at: https://www.urncst.com

likelihood that children with ASD will reach their full potential [20]. Early intervention enables a child displaying ASD symptoms to learn and progress more rapidly. According to recent guidelines, early intervention in ASD can be most beneficial during the time when the diagnosis or suspicion of ASD is confirmed [20]. In the event that such a time period passes by without intervention, it may result in a shortened period for brain growth and development, leaving the brain to rely on underdeveloped structures for carrying out functions beyond episodic memory [20]. It is possible that episodic memory impairments seen in children with ASD may be minimized by supportive learning. Some studies have found evidence that variations in episodic memory performance of children with ASD has been linked to task support [18]. Early researchers have speculated that when episodic retrieval support is low, such as having minimal cues or when information is complex and requires high organization, episodic recollection difficulties in ASD are exacerbated. This hypothesis is supported by the fact that spatial source memory in individuals with ASD is disproportionately enhanced after retrieval support is provided [18].

Episodic memory is heavily used in day-to-day life, education, occupation, and more. Children with ASD often are burdened with symptoms negatively impacting their social, home, and school life [7]. Reducing the deficits on episodic memory would assist in making living with ASD easier for the child in their present and future. The significance and impacts on episodic memory make early interventions crucial to the child's life. Possible interventions for ASD that have been found to minimize episodic memory impairments include the reading and learning of complex words and topics [21]. This activity has been found to have the potential to strengthen neural connections and as a result, mental processing may be accelerated [21]. In addition to the provision of services that have the potential to mitigate episodic memory deficits in children with ASD, it is critical that future studies and interventions consider the social determinants of ASD. There is evidence that existing services that attempt to minimize ASD symptoms such as delayed milestones as well as challenges with verbal and non-verbal communication are underutilized by marginalized communities which further hinders children in these areas from reaching their full cognitive potential [22]. It is integral that future studies and interventions include less represented groups such as children with low-income backgrounds, children of colour, and children from immigrant backgrounds.

\section{Conclusions}

The present study used a literature review to examine the role of connectivity in children with ASD with episodic memory deficits. The results suggest that abnormalities with SC, FC, and EC all play a role in underlying episodic memory impairments in children with ASD. Past research has highlighted the role of connectivity in memory problems found in children with ASD. However, the combination of hypo and hyper connectivity of functional, structural, and effective brain regions must be further explored to determine a diagnostic criterion, treatment, or intervention to minimize the long-lasting effects of episodic memory impairments in childhood.

\section{List of Abbreviations Used}

ASD: autism spectrum disorder

FC: functional connectivity

SC: structural connectivity

EC: effective connectivity

EM: episodic memory

IFG: inferior frontal gyrus

PCC: posterior cingulate cortex

\section{Conflicts of Interest}

The author(s) declare that they have no conflict of interests.

\section{Ethics Approval and/or Participant Consent}

This did not require ethics approval and/or participant consent because this was a literature review.

\section{Authors' Contributions}

XA: made substantial contributions to the design of the study, the collection of data as well as interpretation and analysis of the data, revised the manuscript critically, and gave final approval of the version to be published.

\section{Acknowledgements}

I would like to acknowledge my mentor Amy Yeung who provided technical help, writing assistance and general support throughout this initiative.

\section{Funding}

This study was not funded.

\section{References}

[1] CDC. Diagnostic Criteria [Internet]. Cdc.gov. 2020 [cited 2021 Aug 4]. Available from: https://www.cdc.gov/ncbddd/autism/hcp-dsm.html

[2] CDC. Data \& statistics on autism spectrum disorder [Internet]. Cdc.gov. 2020 [cited 2021 Aug 4]. Available from: https://www.cdc.gov/ncbddd/autism/data.html

[3] Amaral DG, Anderson GM, Bailey A, Bernier R, Bishop S, Blatt G, et al. Gaps in current autism research: The thoughts of the autism research editorial board and associate editors. Autism Research. 2019;12(5):700-14. https://doi.org/10.1002/aur.2101

[4] Rane P, Cochran D, Hodge SM, Haselgrove C, Kennedy DN, Frazier JA. Connectivity in autism: A review of MRI connectivity studies. Harvard Review of Psychiatry. 2015;23(4):223-44. https://doi.org/10.1097/ HRP.0000000000000072 
UNDERGRADUATE RESEARCH IN NATURAL AND CLINICAL SCIENCE AND TECHNOLOGY (URNCST) JOURNAL Read more URNCST Journal articles and submit your own today at: https://www.urncst.com

[5] Rolls ET, Zhou Y, Cheng W, Gilson M, Deco G, Feng J. Effective connectivity in autism. Autism Research. 2020;13(1):32-44. https://doi.org/10.1002/aur.2235

[6] Lind SE, Williams DM, Bowler DM, Peel A. Episodic memory and episodic future thinking impairments in high-functioning autism spectrum disorder: An underlying difficulty with scene construction or selfprojection? Neuropsychology. 2014;28(1):55-67. https://doi.org/10.1037/neu0000005

[7] Dickerson BC, Eichenbaum H. The episodic memory system: Neurocircuitry and disorders. Neuropsychopharmacology. 2010;35(1):86-104. https://doi.org/10.1038/npp.2009.126

[8] Southwick JS, Bigler ED, Froehlich A, DuBray MB, Alexander AL, Lange N, et al. Memory functioning in children and adolescents with autism. Neuropsychology. 2011;25(6):702-10. https://doi.org/ 10.1037/a0024935

[9] Fitzgerald J, Gallagher L, McGrath J. Widespread disrupted white matter microstructure in autism spectrum disorders. Journal of Autism and Development Disorders. 2019;49(7):2664-74. https://doi.org/10.1007/ s10803-016-2803-8

[10] Vogan VM, Morgan BR, Leung RC, Anagnostou E, Doyle-Thomas K, Taylor MJ. Widespread white matter differences in children and adolescents with autism spectrum disorder. Journal of Autism Development Disorders. 2016;46(6):2138-47. https://doi.org/10.1007/ s10803-016-2744-2

[11] Ngo CT, Alm KH, Metoki A, Hampton W, Riggins T, Newcombe NS, et al. White matter structural connectivity and episodic memory in early childhood. Developmental Cognitive Neuroscience. 2017;28:4153. https://doi.org/10.1016/j.den.2017.11.001

[12] Xu S, Li M, Yang C, Fang X, Ye M, Wei L, et al. Altered functional connectivity in children with lowfunction autism spectrum disorders. Frontiers in Neuroscience. 2019;13:806. https://doi.org/10.3389/ fnins.2019.00806

[13] Monk CS, Peltier SJ, Wiggins JL, Weng S-J, Carrasco M, Risi S, et al. Abnormalities of intrinsic functional connectivity in autism spectrum disorders. Neuroimage. 2009;47(2):764-72. https://doi.org/10.1016/j.neuroimage .2009 .04 .069
[14] Barendse EM, Hendriks MP, Jansen JF, Backes WH, Hofman PA, Thoonen G, et al. Working memory deficits in high-functioning adolescents with autism spectrum disorders: Neuropsychological and neuroimaging correlates. Journal of Neurodevelopmental Disorders. 2013;5(1):14. https://doi.org/10.1186/1866-1955-5-14

[15] Ren Y, Nguyen VT, Sonkusare S, Lv J, Pang T, Guo L, et al. Effective connectivity of the anterior hippocampus predicts recollection confidence during natural memory retrieval. Nature Communications. 2018;9(1):4875. https://doi.org/10.1038/s41467-018-07325-4

[16] Bird CM, Burgess N. The hippocampus and memory: Insights from spatial processing. Nature Review Neuroscience. 2008;9(3):182-94. https://doi.org/ $10.1038 / \mathrm{nrn} 2335$

[17] Xu J, Wang H, Zhang L, Xu Z, Li T, Zhou Z, et al. Both hypo-connectivity and hyper-connectivity of the insular subregions associated with severity in children with autism spectrum disorders. Frontiers in Neuroscience. 2018 Apr 11;12. https://doi.org/10.3389/ fnins.2018.00234

[18]Zilli EA, Hasselmo ME. Modeling the role of working memory and episodic memory in behavioral tasks. Hippocampus. 2008;18(2):193-209. https://doi.org/ 10.1002/hipo.20382

[19] Dame Stephanie Shirley. Challenges to autism research. Journal of the Royal Society of Medicine. 2005; 98(11):523-5. https://doi.org/10.1258/jrsm.98.11.523

[20] Berger JM, Rohn TT, Oxford JT. Autism as the early closure of a neuroplastic critical period normally seen in adolescence. Biological Systems Open Access Journal. 2013;1(03). Available from: http://doi.org/ 10.4172/2329-6577.1000118

[21] Ranganath C, Flegal KE, Kelly LL. Can cognitive training improve episodic memory? Neuron. 2011;72(5): 688-91. https://doi.org/10.1016/j.neuron.2011.10.022

[22] Smith KA, Gehricke J-G, Iadarola S, Wolfe A, Kuhlthau KA. Disparities in service use among children with autism: A systematic review. Pediatrics. 2020;145(Suppl 1):S35-46. https://doi.org/10.1542/ peds.2019-1895G

\section{Article Information}

Managing Editor: Jeremy Y. Ng

Peer Reviewers: Amy Yeung, Ricky Chow

Article Dates: Received Aug 04 21; Accepted Oct 25 21; Published Dec 0321

\section{Citation}

Please cite this article as follows:

Ali X. Neural connectivity and episodic memory in autism spectrum disorder: A literature review. URNCST Journal. 2021

Dec 03: 5(12). https://urncst.com/index.php/urncst/article/view/306

DOI Link: https://doi.org/10.26685/urncst.306 
UNDERGRADUATE RESEARCH IN NATURAL AND CLINICAL SCIENCE AND TECHNOLOGY (URNCST) JOURNAL

Read more URNCST Journal articles and submit your own today at: https://www.urncst.com

\section{Copyright}

(C) Xalima Ali. (2021). Published first in the Undergraduate Research in Natural and Clinical Science and Technology (URNCST) Journal. This is an open access article distributed under the terms of the Creative Commons Attribution License (https://creativecommons.org/licenses/by/4.0/), which permits unrestricted use, distribution, and reproduction in any medium, provided the original work, first published in the Undergraduate Research in Natural and Clinical Science and Technology (URNCST) Journal, is properly cited. The complete bibliographic information, a link to the original publication on http://www.urncst.com, as well as this copyright and license information must be included.

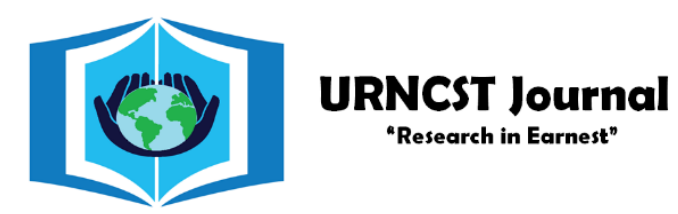

\section{Funded by the Government of Canada}

\section{Canadà̀}

Do you research in earnest? Submit your next undergraduate research article to the URNCST Journal!

| Open Access | Peer-Reviewed | Rapid Turnaround Time | International |

| Broad and Multidisciplinary | Indexed | Innovative | Social Media Promoted |

Pre-submission inquiries? Send us an email at info@urncst.com | Facebook, Twitter and LinkedIn: @URNCST

Submit YOUR manuscript today at https://www.urncst.com! 